eine individuelle Vorhersage auslösender Situationen ermöglichen, sodass der Betroffene gezielter präventive Maßnahmen ergreifen kann.

\section{Kollege Computer macht Digital-} Triage - rein beratend natürlich Wie ein kopfschmerzbezogenes IT-Tool speziell im Bereich Diagnoseunterstützung aussehen kann, illustrierte Professor Miguel Láinez von der Universitätsklinik Valencia am Beispiel der TriageSoftware BonTriage, die von Neurologen der Universitäten Stanford und Los Angeles/Südkalifornien entwickelt wurde. Sie kann online oder als App für Mobilgeräte von Patienten bereits heute genutzt werden, bisher aber nur auf Englisch.

BonTriage ist letztlich eine Art Anamneseprogramm, und zwar ein ziemlich umfangreiches. Patienten benötigen im
Schnitt 30 bis 60 Minuten, um es auszufüllen. Die Software fragt Symptome detailliert ab, evaluiert die Medikationshistorie und viele andere Aspekte, die zu einer umfangreichen ärztlichen Kopfschmerzanamnese dazugehören, die im eng getakteten Alltag aber oft unter den Tisch fallen.

Aus all diesen Informationen generiert BonTriage unter Zuhilfenahme von Entscheidungsbäumen und $\mathrm{Ma}$ schinenlernalgorithmen einen „Kopfschmerzreport“, den der Patient seinem Arzt mailen oder auch ausdrucken und mitbringen kann. Er enthält die übersichtlich aufbereitete Anamnese, weist auf Warnzeichen für sekundären Kopfschmerz hin und bietet eine Art Differenzialdiagnose an, zusammen mit einer Einschätzung, die von den möglichen Diagnosen die wahrscheinlichste sein könnte.
In Barcelona hat Láinez erste Erfahrungen mit BonTriage gesammelt. Die Software liefere zwar nicht in allen Fällen die korrekte Verdachtsdiagnose, funktioniere aber insgesamt sehr gut. Insbesondere hob er hervor, dass sieben Cluster-Kopfschmerzpatienten korrekt identifiziert wurden, und dass die Software in mehreren Fällen auf einen möglichen medikamenteninduzierten Kopfschmerz sowie auf mit Kopfschmerz in Zusammenhang stehende Unfälle beziehungsweise Verletzungen hingewiesen habe. „Das Programm ist nicht besser als Experten, aber es ist besser als die meisten Allgemeinmediziner. Und vor allem kann es den Ärzten viel Zeit sparen“, so das Fazit von Láinez.

Philipp Grätzel von Grätz

Session Controversy 1 "Controversies in headache and Prakinson's Disease"

3. EAN-Kongress, 24.-27.6.2017, Amsterdam

\title{
Zu wenig Vitamin D bei RLS?
}

\section{Möglicherweise ist der Vitamin-D-Spiegel nicht nur bei Multipler Sklerose, sondern auch beim Restless-Legs-Syndrom (RLS) relevant. Einer Studie zufolge haben Patienten mit niedrigen Werten des Vitamins häufig starke Beschwerden.}

\footnotetext{
A uch Neurologen haben mittlerweile das Vitamin D entdeckt: So gibt es nicht nur Hinweise auf einen relevanten Einfluss in der MS-Pathologie, möglicherweise kann Calcitriol auch den Verlauf von Störungen im dopaminergen System beeinflussen. Nach Resultaten von Tierversuchen fördert Vitamin D die nigrostriatale Dopamintransmission, ein Mangel könnte daher Krankheiten wie RLS und Parkinson begünstigen.

In kleineren nicht kontrollierten Studien wurden erniedrigte Vitamin-DWerte bei RLS-Patienten gemessen, auch scheinen die RLS-Symptome bei Betroffenen mit Vitamin-D-Mangel durch eine Supplementierung etwas zurückzugehen, erläuterte Dr. Ambra Stefani, Universität Innsbruck, beim EAN-Kongress in Amsterdam. Ihre Arbeitsgruppe hat daher selbst bei 107 RLS-Patienten sowie 107 gleichaltrigen Personen ohne RLS nachgemessen. Das Alter der Studienteilnehmer lag im Schnitt bei 50
}

Jahren, in beiden Gruppen waren etwas mehr als die Hälfte Frauen. Ausgeschlossen von der Studienteilnahme wurden Personen mit Vitamin-D-Supplementierung sowie Patienten mit einer Häufung von RLS-Erkrankungen in der Familie - hier gehen die Studienärzte davon aus, dass eher die Gene und weniger die Vitamin-D-Spiegel relevant sind. Bei allen Teilnehmern bestimmten die Ärzte die 25-Hydroxy-Vitamin-D3-Konzentrationen. Werte unter $20 \mathrm{ng} / \mathrm{ml}$ gelten als Mangel.

\section{Gehäuft Vitamin-D-Mangel bei RLS}

Wie sich zeigte, unterschieden sich die Vitamin-D-Werte im Schnitt nicht signifikant zwischen Personen mit und ohne RLS. Allerdings wurde ein Vitamin-D-Mangel bei neun Patienten mit RLS festgestellt, in der Kontrollgruppe war nur eine Person betroffen. Patienten mit Vitamin-D-Mangel hatten zudem schwerere Symptome - der Wert auf der internationalen RLS Severity Scale (IRLS) war signifikant höher. Insgesamt gab es aber einen eher schwachen $\mathrm{Zu}$ sammenhang zwischen IRLS-Werten und Vitamin-D-Spiegeln. Dieser verstärkte sich jedoch, wenn nur die Patienten mit spät beginnendem RLS berücksichtigt wurden. Bei solchen Patienten wird davon ausgegangen, dass Umweltfaktoren relevanter sind als bei früh beginnendem RLS. Bei ihnen konnten die Neurologen aus Innsbruck immerhin eine moderate Korrelation beobachten, die Symptome waren umso ausgeprägter, je niedriger die Vitamin-D-Spiegel lagen, erläuterte Stefani.

Auf welche Weise ein Vitamin-DMangel die Entstehung eines RLS oder dessen Ausprägung beeinflusst, ist noch weitgehend unklar. Stefani nannte Arbeiten, die einen Zusammenhang mit dem Eisenstoffwechsel nahelegen. Offenbar sorgt zu wenig Vitamin D für einen Eisenmangel im Gehirn, der wiederum die dopaminerge Transmission stört. Letztlich müssen aber weitere klinische Studien zeigen, ob dieser Mechanismus für RLS relevant ist.

Thomas Müller

Oral Session: Sleep Disorders.

3. EAN-Kongress, 24.-27.6.2017, Amsterdam 